\title{
Lattice Structure of Generalized Intuitionistic Fuzzy Soft Sets
}

\author{
Jin Han Park \\ Department of Applied Mathematics, Pukyong National University, Busan 608-737, Korea
}

\begin{abstract}
Park et al. introduced the concept of generalized intuitionistic fuzzy soft sets, which can be seen as an effective mathematical tool to deal with uncertainties. In this paper, we introduce new operations such as restricted union and restricted intersection and study their basic properties, and deal with the algebraic structure of generalized intuitionistic fuzzy soft sets. The lattice structures of generalized intuitionistic fuzzy soft sets are constructed.
\end{abstract}

Key Words: Generalized intuitionistic fuzzy soft sets, Restricted union, Restricted intersection, Necessity and possibility operations.

\section{Introduction}

Molodtsov [1] introduced soft sets as a mathematical tool for dealing with uncertainties which is free from the inherent difficulties that probability theory, fuzzy set theory, vague set theory and rough set theory have (see [1]). Since the soft set theory offers mathematical tool for dealing with uncertain, fuzzy and not clearly defined objects, it has a rich potential for applications to problems in real life situation. Lots of studies on this issue have been extended to fuzzy environment and its extended environments [1-13]. Recently, Park et al. [14] presented the concept of the generalized intuitionistic fuzzy soft sets by combining the generalized intuitionistic fuzzy sets [15] and soft set models. Park et al. [10] presented some results on an application of

접수일자 : 2013년 11월 19일

심사(수정)일자 : 2013년 12월 10일

게재확정일자 : 2013년 12월 11일

This is an Open-Access article distributed under the terms of the Creative Commons Attribution Non-Commercial License http: //creativecommons.org/licenses/by-nc/3.0/ which permits unrestricted non-commercial use, distribution, and reproduction in any medium, provided the original work is properly cited. generalized intuitionistic fuzzy soft sets in decision making problems. In [16], Park tried to find an answer to the question how the logic operations and their interrelations between each other correspond to generalized intuitionistic fuzzy set operations. In this paper, we make a theoretical study of the generalized intuitionistic fuzzy soft set theory and concentrate on the algebraic structure of generalized intuitionistic fuzzy soft sets. The lattice structures of generalized intuitionistic fuzzy soft sets are constructed.

\section{Preliminaries}

This section presents a review of some fundamental notions of generalized intuitionistic fuzzy soft sets. See [10, 14, 16] for further details and background.

Definition 2.1. [14] Let $U$ be an initial universe and $E$ be a set of parameters. $\mathcal{G} \mathcal{I} \mathcal{F}(U)$ denotes the set of all generalized intuitionistic fuzzy sets of $U$. Let $A \subseteq E$. A pair $\langle F, A\rangle$ is a generalized intuitionistic fuzzy soft set over $U$, where $F$ is a mapping given by $F: A \rightarrow \mathcal{G I F}(U)$.

In other words, a generalized intuitionistic fuzzy soft set is a parameterized family of generalized intuitionistic fuzzy subsets of $U$ and thus its universe is the set of all generalized intuitionistic fuzzy sets of $U$, i.e., $\mathcal{G} \mathcal{I} \mathcal{F}(U)$. 
Definition 2.2. [14] Let $\langle F, A\rangle$ and $\langle G, B\rangle$ be two generalized intuitionistic fuzzy soft sets over $U$. Then $\langle F, A\rangle$ is said to be a generalized intuitionistic fuzzy soft subset of $\langle G, B\rangle$ if

(1) $A \subseteq B$;

(2) for any $\varepsilon \in A, F(\varepsilon)$ is a generalized fuzzy subset of $G(\varepsilon)$, that is, for all $x \in U$ and $\varepsilon \in A, \mu_{F(\varepsilon)}(x) \leq$ $\mu_{G(\varepsilon)}(x)$ and $\nu_{F(\varepsilon)}(x) \geq \nu_{G(\varepsilon)}(x)$.

This relationship is denoted by $\langle F, A\rangle \sqsubseteq\langle G, B\rangle$. Similarly, $\langle F, A\rangle$ is said to be a generalized intuitionistic fuzzy soft superset of $\langle G, B\rangle$, if $\langle G, B\rangle$ is called a generalized intuitionistic fuzzy soft subset of $\langle F, A\rangle$. We denote it by $\langle F, A\rangle \sqsupseteq\langle G, B\rangle$.

Definition 2.3. [14 Let $E=\left\{\varepsilon_{1}, \varepsilon_{2}, \ldots, \varepsilon_{n}\right\}$ be a set of parameters. The not of $E$, denoted by $\rceil E$, is defined by \rceil $E=\left\{\neg \varepsilon_{1}, \neg \varepsilon_{2}, \ldots, \neg \varepsilon_{n}\right\}$, where $\neg \varepsilon_{i}=$ not $\varepsilon_{i}$.

Definition 2.4. [14] The complement of a generalized intuitionistic fuzzy soft set $\langle F, A\rangle$, denoted by $\langle F, A\rangle^{c}$, is defined by $\left.\langle F, A\rangle^{c}=\left\langle F^{c},\right\rceil A\right\rangle$, where $\left.F^{c}:\right\rceil A \rightarrow \mathcal{G I F}(U)$ is a mapping given by $F^{c}(\varepsilon)=\left\langle x, \nu_{F(\neg \varepsilon)}(x), \mu_{F(\neg \varepsilon)}(x)\right\rangle$ for all $x \in U$ and $\varepsilon \in\rceil A$.

Definition 2.5. [14] A generalized intuitionistic fuzzy soft set $\langle F, A\rangle$ is said to be a null generalized intuitionistic fuzzy soft set, denoted by $\Phi_{A}$, if for any $\varepsilon \in A$, $\mu_{F(\varepsilon)}(x)=0$ and $\nu_{F(\varepsilon)}(x)=1$, for all $x \in U$.

Definition 2.6. [14] A generalized intuitionistic fuzzy soft set $\langle F, A\rangle$ is said to be an absolute generalized intuitionistic fuzzy soft set, denoted by $\Sigma_{A}$, if for any $\varepsilon \in A, \mu_{F(\varepsilon)}(x)=$ 1 and $\nu_{F(\varepsilon)}(x)=0$, for all $x \in U$.

\section{Lattice structure of generalized intuitionistic fuzzy soft sets}

Algebraic structures play a fundamental role in many fields of mathematics. In this section, we discuss the lattice structure of generalized intuitionistic fuzzy soft sets.

Definition 3.1. [16] The union of two generalized intuitionistic fuzzy soft sets $\langle F, A\rangle$ and $\langle G, B\rangle$ over a universe $U$ is a generalized intuitionistic fuzzy soft set $\langle H, C\rangle$, where $C=A \cup B$, and for all $\varepsilon \in C$ and $x \in U$,

$$
\begin{gathered}
\mu_{H(\varepsilon)}(x)= \begin{cases}\mu_{F(\varepsilon)}(x), & \text { if } \varepsilon \in A-B, \\
\mu_{G(\varepsilon)}(x), & \text { if } \varepsilon \in B-A, \\
\max \left\{\mu_{F(\varepsilon)}(x), \mu_{G(\varepsilon)}(x)\right\}, & \text { if } \varepsilon \in A \cap B,\end{cases} \\
\nu_{H(\varepsilon)}(x)= \begin{cases}\nu_{F(\varepsilon)}(x), & \text { if } \varepsilon \in A-B, \\
\nu_{G(\varepsilon)}(x), & \text { if } \varepsilon \in B-A, \\
\min \left\{\nu_{F(\varepsilon)}(x), \nu_{G(\varepsilon)}(x)\right\}, & \text { if } \varepsilon \in A \cap B .\end{cases}
\end{gathered}
$$

We denoted it by $\langle F, A\rangle \sqcup\langle G, B\rangle=\langle H, C\rangle$.

Definition 3.2. [16] The intersection of two generalized intuitionistic fuzzy soft sets $\langle F, A\rangle$ and $\langle G, B\rangle$ over a universe $U$ is a generalized intuitionistic fuzzy soft set $\langle H, C\rangle$, where $C=A \cup B$, and for all $\varepsilon \in C$ and $x \in U$,

$\mu_{H(\varepsilon)}(x)= \begin{cases}\mu_{F(\varepsilon)}(x), & \text { if } \varepsilon \in A-B, \\ \mu_{G(\varepsilon)}(x), & \text { if } \varepsilon \in B-A, \\ \min \left\{\mu_{F(\varepsilon)}(x), \mu_{G(\varepsilon)}(x)\right\}, & \text { if } \varepsilon \in A \cap B,\end{cases}$
$\nu_{H(\varepsilon)}(x)= \begin{cases}\nu_{F(\varepsilon)}(x), & \text { if } \varepsilon \in A-B, \\ \nu_{G(\varepsilon)}(x), & \text { if } \varepsilon \in B-A, \\ \max \left\{\nu_{F(\varepsilon)}(x), \nu_{G(\varepsilon)}(x)\right\}, & \text { if } \varepsilon \in A \cap B .\end{cases}$

We denoted it by $\langle F, A\rangle \sqcap\langle G, B\rangle=\langle H, C\rangle$.

Theorem 3.3. [16] Let $E$ be a set of parameters and $A \subset$ $E$. If $\langle F, A\rangle$ and $\langle F, E\rangle$ are two generalized intuitionistic fuzzy soft sets over $U$, then the following properties hold:
(1) $\langle F, A\rangle \sqcup\langle F, A\rangle=\langle F, A\rangle$;
(2) $\langle F, A\rangle \sqcap\langle F, A\rangle=\langle F, A\rangle$;
(3) $\langle F, A\rangle \sqcup\langle G, B\rangle=\langle G, B\rangle \sqcup\langle F, A\rangle$;
(4) $\langle F, A\rangle \sqcap\langle G, B\rangle=\langle G, B\rangle \sqcap\langle F, A\rangle$;
(5) $(\langle F, A\rangle \sqcup\langle G, B\rangle) \sqcup\langle H, C\rangle=\langle F, A\rangle \sqcup(\langle G, B\rangle \sqcup$ $\langle H, C\rangle)$;

(6) $(\langle F, A\rangle \sqcap\langle G, B\rangle) \sqcap\langle H, C\rangle=\langle F, A\rangle \sqcap(\langle G, B\rangle \sqcap$ $\langle H, C\rangle)$.

Definition 3.4. The restricted union of two generalized intuitionistic fuzzy soft sets $\langle F, A\rangle$ and $\langle G, B\rangle$ over a universe $U$, denoted by $\langle F, A\rangle \tilde{\cup}\langle G, B\rangle$, is a generalized intuitionistic fuzzy soft set $\langle H, C\rangle$, where $C=A \cap B$, and for all $\varepsilon \in C$ and $x \in U$,

$$
\begin{aligned}
& \mu_{H(\varepsilon)}(x)=\max \left\{\mu_{F(\varepsilon)}(x), \mu_{G(\varepsilon)}(x)\right\} \\
& \nu_{H(\varepsilon)}(x)=\min \left\{\nu_{F(\varepsilon)}(x), \nu_{G(\varepsilon)}(x)\right\}
\end{aligned}
$$

Definition 3.5. The restricted intersection of two generalized intuitionistic fuzzy soft sets $\langle F, A\rangle$ and $\langle G, B\rangle$ over 
a universe $U$, denoted by $\langle F, A\rangle \tilde{\cap}\langle G, B\rangle$, is a generalized intuitionistic fuzzy soft set $\langle H, C\rangle$, where $C=A \cap B$, and for all $\varepsilon \in C$ and $x \in U$,

$$
\begin{aligned}
& \mu_{H(\varepsilon)}(x)=\min \left\{\mu_{F(\varepsilon)}(x), \mu_{G(\varepsilon)}(x)\right\}, \\
& \nu_{H(\varepsilon)}(x)=\max \left\{\nu_{F(\varepsilon)}(x), \nu_{G(\varepsilon)}(x)\right\} .
\end{aligned}
$$

Theorem 3.6. Let $\langle F, A\rangle,\langle G, B\rangle$ and $\langle H, C\rangle$ be three generalized intuitionistic fuzzy soft sets over the same universe $U$. Then

(1) $\langle F, A\rangle \tilde{\cup}\langle F, A\rangle=\langle F, A\rangle$.

(2) $\langle F, A\rangle \tilde{\cup}\langle G, B\rangle=\langle G, B\rangle \tilde{\cup}\langle F, A\rangle$.

(3) $(\langle F, A\rangle \tilde{\cup}\langle G, B\rangle) \tilde{\cup}\langle H, C\rangle=\langle F, A\rangle \tilde{\cup}(\langle G, B\rangle \tilde{\cup}\langle H$, $C\rangle)$.

Theorem 3.7. Let $\langle F, A\rangle,\langle G, B\rangle$ and $\langle H, C\rangle$ be three generalized intuitionistic fuzzy soft sets over the same universe $U$. Then

(1) $\langle F, A\rangle \tilde{\cap}\langle F, A\rangle=\langle F, A\rangle$.

(2) $\langle F, A\rangle \tilde{\cap}\langle G, B\rangle=\langle G, B\rangle \tilde{\cap}\langle F, A\rangle$.

(3) $(\langle F, A\rangle \tilde{\cap}\langle G, B\rangle) \tilde{\cap}\langle H, C\rangle=\langle F, A\rangle \tilde{\cap}(\langle G, B\rangle \tilde{\cap}\langle H$, $C\rangle)$.

The following theorem shows that the absorption law with respect to the operations $\tilde{\cup}$ and $\tilde{\cap}$ holds.

Theorem 3.8. Let $\langle F, A\rangle$ and $\langle G, B\rangle$ be two generalized intuitionistic fuzzy soft sets over the same universe $U$. Then

(1) $(\langle F, A\rangle \sqcup\langle G, B\rangle) \tilde{\cap}\langle F, A\rangle=\langle F, A\rangle$.

(2) $(\langle F, A\rangle \tilde{\cap}\langle G, B\rangle) \sqcup\langle F, A\rangle=\langle F, A\rangle$.

Proof. (1) Suppose that $\langle F, A\rangle \sqcup\langle G, B\rangle=\langle H, A \cup B\rangle$ and $(\langle F, A\rangle \sqcup\langle G, B\rangle) \tilde{\cap}\langle F, A\rangle=\langle K,(A \cup B) \cap A\rangle=\langle K, A\rangle$. For all $\varepsilon \in A$ and $x \in U$,

(a) if $\varepsilon \in B$, then

$$
\begin{aligned}
\mu_{K(\varepsilon)}(x) & =\min \left\{\mu_{H(\varepsilon)}(x), \mu_{F(\varepsilon)}(x)\right\} \\
& =\min \left\{\max \left\{\mu_{F(\varepsilon)}(x), \mu_{G(\varepsilon)}(x)\right\}, \mu_{F(\varepsilon)}(x)\right\} \\
& =\mu_{F(\varepsilon)}(x)
\end{aligned}
$$

and

$$
\begin{aligned}
\nu_{K(\varepsilon)}(x) & =\max \left\{\nu_{H(\varepsilon)}(x), \nu_{F(\varepsilon)}(x)\right\} \\
& =\max \left\{\min \left\{\nu_{F(\varepsilon)}(x), \nu_{G(\varepsilon)}(x)\right\}, \nu_{F(\varepsilon)}(x)\right\} \\
& =\nu_{F(\varepsilon)}(x) .
\end{aligned}
$$

(b) if $\varepsilon \notin B$, then

$$
\begin{aligned}
\mu_{K(\varepsilon)}(x) & =\min \left\{\mu_{H(\varepsilon)}(x), \mu_{F(\varepsilon)}(x)\right\} \\
& =\min \left\{\mu_{F(\varepsilon)}(x) \mu_{F(\varepsilon)}(x)\right\} \\
& =\mu_{F(\varepsilon)}(x)
\end{aligned}
$$

and

$$
\begin{aligned}
\nu_{K(\varepsilon)}(x) & =\max \left\{\nu_{H(\varepsilon)}(x), \nu_{F(\varepsilon)}(x)\right\} \\
& =\max \left\{\nu_{F(\varepsilon)}(x) \nu_{F(\varepsilon)}(x)\right\} \\
& =\nu_{F(\varepsilon)}(x) .
\end{aligned}
$$

Hence $(\langle F, A\rangle \sqcup\langle G, B\rangle) \tilde{\cap}\langle F, A\rangle=\langle F, A\rangle$.

(2) Suppose that $\langle F, A\rangle \tilde{\cap}\langle G, B\rangle=\langle H, A \cap B\rangle$ and $(\langle F, A\rangle \tilde{\cap}\langle G, B\rangle) \sqcup\langle F, A\rangle=\langle K,(A \cap B) \cup A\rangle=\langle K, A\rangle$.

For all $\varepsilon \in A$ and $x \in U$,

(a) if $\varepsilon \in B$, then

$$
\begin{aligned}
\mu_{K(\varepsilon)}(x) & =\max \left\{\mu_{H(\varepsilon)}(x), \mu_{F(\varepsilon)}(x)\right\} \\
& =\max \left\{\min \left\{\mu_{F(\varepsilon)}(x), \mu_{G(\varepsilon)}(x)\right\}, \mu_{F(\varepsilon)}(x)\right\} \\
& =\mu_{F(\varepsilon)}(x)
\end{aligned}
$$

and

$$
\begin{aligned}
\nu_{K(\varepsilon)}(x) & =\min \left\{\nu_{H(\varepsilon)}(x), \nu_{F(\varepsilon)}(x)\right\} \\
& =\min \left\{\max \left\{\nu_{F(\varepsilon)}(x), \nu_{G(\varepsilon)}(x)\right\}, \nu_{F(\varepsilon)}(x)\right\} \\
& =\nu_{F(\varepsilon)}(x) .
\end{aligned}
$$

(b) if $\varepsilon \notin B$, then $\varepsilon \notin A \cap B$. It follows that $\mu_{K(\varepsilon)}(x)=$ $\mu_{F(\varepsilon)}(x)$ and $\nu_{K(\varepsilon)}(x)=\nu_{F(\varepsilon)}(x)$.

Hence $(\langle F, A\rangle \tilde{\cap}\langle G, B\rangle) \sqcup\langle F, A\rangle=\langle F, A\rangle$.

Now, we denote by $\mathcal{G I \mathcal { F }} \mathcal{S}(U, E)$ the set of all generalized intuitionistic fuzzy soft sets over the universe $U$ and the parameter set $E$, that is

$\mathcal{G I F \mathcal { S }}(U, E)=\{\langle F, A\rangle \mid A \subseteq E, F: A \rightarrow \mathcal{G} \mathcal{I} \mathcal{F}(U)\}$

Theorem 3.9. (1) $(\mathcal{G I F \mathcal { F }}(U, E), \sqcup, \tilde{\cap})$ is a distributive lattice.

(2) Let $\leq_{1}$ be the ordering relation in lattice $(\mathcal{G} \mathcal{I} \mathcal{F} \mathcal{S}(U, E), \sqcup, \tilde{\cap})$ and $\langle F, A\rangle,\langle G, B\rangle \in \mathcal{G} \mathcal{I} \mathcal{F} \mathcal{S}(U, E)$. Then $\langle F, A\rangle \leq_{1}\langle G, B\rangle$ if and only if $A \subseteq B$, and $\mu_{F(\varepsilon)}(x) \leq \mu_{G(\varepsilon)}(x)$ and $\nu_{F(\varepsilon)}(x) \geq \nu_{G(\varepsilon)}(x)$ for all $\varepsilon \in A$ and $x \in U$. 
Proof. (1) By Theorems 3.3, 3.7 and 3.8, $(\mathcal{G I F S}(U, E)$, $\sqcup, \tilde{\cap})$ is a lattice. We prove that the following distributive law

$$
\begin{aligned}
& \langle F, A\rangle \sqcup(\langle G, B\rangle \tilde{\cap}\langle H, C\rangle) \\
& \quad=(\langle F, A\rangle \sqcup\langle G, B\rangle) \tilde{\cap}(\langle F, A\rangle \sqcup\langle H, C\rangle)
\end{aligned}
$$

holds for all $\langle F, A\rangle,\langle G, B\rangle,\langle H, C\rangle \in \mathcal{G} \mathcal{I F} \mathcal{S}(U, E)$.

Suppose that $\langle F, A\rangle \sqcup(\langle G, B\rangle \tilde{\cap}\langle H, C\rangle)=\langle K, A \cup(B \cap$ $C)\rangle$ and $(\langle F, A\rangle \sqcup\langle G, B\rangle) \tilde{\cap}(\langle F, A\rangle \sqcup\langle H, C\rangle)=\langle L,(A \cup$ $B) \cap(A \cup C)\rangle=\langle L, A \cup(B \cap C)\rangle$.

For all $\varepsilon \in A \cup(B \cap C)$ and $x \in U$,

(a) if $\varepsilon \notin A$, then $\varepsilon \in B$ and $\varepsilon \in C$, then

$$
\begin{aligned}
\mu_{K(\varepsilon)}(x) & =\min \left\{\mu_{G(\varepsilon)}(x), \mu_{H(\varepsilon)}(x)\right\} \\
& =\mu_{L(\varepsilon)}(x)
\end{aligned}
$$

and

$$
\begin{aligned}
\nu_{K(\varepsilon)}(x) & =\max \left\{\nu_{G(\varepsilon)}(x), \nu_{H(\varepsilon)}(x)\right\} \\
& =\nu_{L(\varepsilon)}(x) .
\end{aligned}
$$

(b) if $\varepsilon \in A, \varepsilon \notin B, \varepsilon \notin C$, then

$$
\begin{aligned}
\mu_{K(\varepsilon)}(x) & =\mu_{F(e)}(x) \\
& =\min \left\{\mu_{F(\varepsilon)}(x), \mu_{F(\varepsilon)}(x)\right\} \\
& =\mu_{L(\varepsilon)}(x)
\end{aligned}
$$

and

$$
\begin{aligned}
\nu_{K(\varepsilon)}(x) & =\nu_{F(e)}(x) \\
& =\max \left\{\nu_{F(\varepsilon)}(x), \nu_{F(\varepsilon)}(x)\right\} \\
& =\nu_{L(\varepsilon)}(x) .
\end{aligned}
$$

(c) if $\varepsilon \in A, \varepsilon \in B, \varepsilon \notin C$, then

$$
\begin{aligned}
\mu_{K(\varepsilon)}(x) & =\mu_{F(e)}(x) \\
& =\min \left\{\max \left\{\mu_{F(\varepsilon)}(x), \mu_{G(\varepsilon)}(x)\right\}, \mu_{F(\varepsilon)}(x)\right\} \\
& =\mu_{L(\varepsilon)}(x)
\end{aligned}
$$

and

$$
\begin{aligned}
\nu_{K(\varepsilon)}(x) & =\nu_{F(e)}(x) \\
& =\max \left\{\min \left\{\nu_{F(\varepsilon)}(x), \nu_{G(\varepsilon)}(x)\right\}, \nu_{F(\varepsilon)}(x)\right\} \\
& =\nu_{L(\varepsilon)}(x) .
\end{aligned}
$$

(d) if $\varepsilon \in A, \varepsilon \notin B, \varepsilon \in C$, then

$$
\begin{aligned}
\mu_{K(\varepsilon)}(x) & =\mu_{F(e)}(x) \\
& =\min \left\{\mu_{F(\varepsilon)}(x), \max \left\{\mu_{F(\varepsilon)}(x), \mu_{H(\varepsilon)}(x)\right\}\right\} \\
& =\mu_{L(\varepsilon)}(x)
\end{aligned}
$$

and

$$
\begin{aligned}
\nu_{K(\varepsilon)}(x) & =\nu_{F(e)}(x) \\
& =\max \left\{\nu_{F(\varepsilon)}(x), \min \left\{\nu_{F(\varepsilon)}(x), \nu_{H(\varepsilon)}(x)\right\}\right\} \\
& =\nu_{L(\varepsilon)}(x) .
\end{aligned}
$$

(e) if $\varepsilon \in A, \varepsilon \in B, \varepsilon \in C$, then

$$
\begin{aligned}
\mu_{K(\varepsilon)}(x)= & \max \left\{\mu_{F(\varepsilon)}(x), \min \left\{\mu_{G(\varepsilon)}(x), \mu_{H(\varepsilon)}(x)\right\}\right\} \\
= & \min \left\{\max \left\{\mu_{F(\varepsilon)}(x), \mu_{G(\varepsilon)}(x)\right\},\right. \\
& \left.\quad \max \left\{\mu_{F(\varepsilon)}(x), \mu_{H(\varepsilon)}(x)\right\}\right\} \\
= & \mu_{L(\varepsilon)}(x)
\end{aligned}
$$

and

$$
\begin{aligned}
\nu_{K(\varepsilon)}(x)= & \min \left\{\nu_{F(\varepsilon)}(x), \max \left\{\nu_{G(\varepsilon)}(x), \nu_{H(\varepsilon)}(x)\right\}\right\} \\
= & \max \left\{\min \left\{\nu_{F(\varepsilon)}(x), \nu_{G(\varepsilon)}(x)\right\}\right. \\
& \left.\quad \min \left\{\nu_{F(\varepsilon)}(x), \nu_{H(\varepsilon)}(x)\right\}\right\} \\
= & \nu_{L(\varepsilon)}(x)
\end{aligned}
$$

Hence $\langle F, A\rangle \sqcup(\langle G, B\rangle \tilde{\cap}\langle H, C\rangle)=(\langle F, A\rangle \sqcup\langle G, B\rangle)$ $\tilde{\cap}(\langle F, A\rangle \sqcup\langle H, C\rangle)$.

(2) Suppose that $\langle F, A\rangle \leq_{1}\langle G, B\rangle$. Then $\langle F, A\rangle \sqcup$ $\langle G, B\rangle=\langle G, B\rangle$. So, by definition, we have $A \cup$ $B=B$ and hence $A \subseteq B$. For all $\varepsilon \in A$ and $x \in U$, since $\max \left\{\mu_{F(\varepsilon)}(x), \mu_{G(\varepsilon)}(x)\right\}=\mu_{G(\varepsilon)}(x)$ and $\min \left\{\nu_{F(\varepsilon)}(x), \nu_{G(\varepsilon)}(x)\right\}=\nu_{G(\varepsilon)}(x)$, we have $\mu_{F(\varepsilon)}(x) \leq \mu_{G(\varepsilon)}(x)$ and $\nu_{F(\varepsilon)}(x) \geq \nu_{G(\varepsilon)}(x)$.

Conversely, suppose that $A \subseteq B$, and $\mu_{F(\varepsilon)}(x) \leq$ $\mu_{G(\varepsilon)}(x)$ and $\nu_{F(\varepsilon)}(x) \geq \nu_{G(\varepsilon)}(x)$ for all $\varepsilon \in A$ and $x \in U$. It is trivial to verify that $\langle F, A\rangle \sqcup\langle G, B\rangle=\langle G, B\rangle$ and hence $\langle F, A\rangle \leq_{1}\langle G, B\rangle$.

Clearly, $(\mathcal{G} \mathcal{I} \mathcal{F} \mathcal{S}(U, E), \sqcup, \tilde{n})$ is bounded lattice, $\Sigma_{E}$ and $\Phi_{\emptyset}$ are the upper bound and lower bound, respectively. We consider generalized intuitionistic fuzzy soft sets over definite parameter set. Let $A \subseteq E$ and

$$
\mathcal{G I F S}_{A}=\{\langle F, A\rangle \mid F: A \rightarrow \mathcal{G I F}(U)\}
$$

be the set of generalized intuitionistic fuzzy soft sets over the unverse $U$ and the parameter set $A$. It is easy to show that $\langle F, A\rangle \sqcup\langle G, A\rangle),\langle F, A\rangle \tilde{\cap}\langle G, A\rangle) \in \mathcal{G I F}^{\mathcal{F}} \mathcal{S}_{A}$.

Corollary 3.10. $\mathcal{G I F S}_{A}$ is sublattice of $(\mathcal{G I F} \mathcal{S}(U, E)$, $\sqcup, \tilde{\cap})$. 
In what follows, we consider another lattice structure of generalized intuitionistic fuzzy soft sets.

Theorem 3.11. Let $\langle F, A\rangle$ and $\langle G, B\rangle$ be two generalized intuitionistic fuzzy soft sets over the same universe $U$. Then

(1) $(\langle F, A\rangle \tilde{\cup}\langle G, B\rangle) \sqcap\langle F, A\rangle=\langle F, A\rangle$.

(2) $(\langle F, A\rangle \sqcap\langle G, B\rangle) \tilde{\cup}\langle F, A\rangle=\langle F, A\rangle$.

Proof. (1) Suppose that $\langle F, A\rangle \tilde{\cup}\langle G, B\rangle=\langle H, A \cap B\rangle$ and $(\langle F, A\rangle \tilde{\cup}\langle G, B\rangle) \sqcap\langle F, A\rangle=\langle K,(A \cap B) \cup A\rangle=\langle K, A\rangle$. For all $\varepsilon \in A$ and $x \in U$,

(a) if $\varepsilon \in B$, then $\varepsilon \in A \cap B$ and so

$$
\begin{aligned}
\mu_{K(\varepsilon)}(x) & =\min \left\{\mu_{H(\varepsilon)}(x), \mu_{F(\varepsilon)}(x)\right\} \\
& =\min \left\{\max \left\{\mu_{F(\varepsilon)}(x), \mu_{G(\varepsilon)}(x)\right\}, \mu_{F(\varepsilon)}(x)\right\} \\
& =\mu_{F(\varepsilon)}(x)
\end{aligned}
$$

and

$$
\begin{aligned}
\nu_{K(\varepsilon)}(x) & =\max \left\{\nu_{H(\varepsilon)}(x), \nu_{F(\varepsilon)}(x)\right\} \\
& =\max \left\{\min \left\{\nu_{F(\varepsilon)}(x), \nu_{G(\varepsilon)}(x)\right\}, \nu_{F(\varepsilon)}(x)\right\} \\
& =\nu_{F(\varepsilon)}(x) .
\end{aligned}
$$

(b) if $\varepsilon \notin B$, then $\varepsilon \notin A \cap B$ and so $\mu_{K(\varepsilon)}(x)=$ $\mu_{F(\varepsilon)}(x)$ and $\nu_{K(\varepsilon)}(x)=\nu_{F(\varepsilon)}(x)$.

Hence $(\langle F, A\rangle \tilde{\cup}\langle G, B\rangle) \sqcap\langle F, A\rangle=\langle F, A\rangle$.

(2) Suppose that $\langle F, A\rangle \sqcap\langle G, B\rangle=\langle H, A \cup B\rangle$ and $(\langle F, A\rangle \sqcap\langle G, B\rangle) \tilde{\cup}\langle F, A\rangle=\langle K,(A \cup B) \cap A\rangle=\langle K, A\rangle$. For all $\varepsilon \in A$ and $x \in U$,

(a) if $\varepsilon \in B$, then $\varepsilon \in A \cap B$ and so

$$
\begin{aligned}
\mu_{K(\varepsilon)}(x) & =\max \left\{\mu_{H(\varepsilon)}(x), \mu_{F(\varepsilon)}(x)\right\} \\
& =\max \left\{\min \left\{\mu_{F(\varepsilon)}(x), \mu_{G(\varepsilon)}(x)\right\}, \mu_{F(\varepsilon)}(x)\right\} \\
& =\mu_{F(\varepsilon)}(x)
\end{aligned}
$$

and

$$
\begin{aligned}
\nu_{K(\varepsilon)}(x) & =\min \left\{\nu_{H(\varepsilon)}(x), \nu_{F(\varepsilon)}(x)\right\} \\
& =\min \left\{\max \left\{\nu_{F(\varepsilon)}(x), \nu_{G(\varepsilon)}(x)\right\}, \nu_{F(\varepsilon)}(x)\right\} \\
& =\nu_{F(\varepsilon)}(x) .
\end{aligned}
$$

(b) if $\varepsilon \notin B$, then

$$
\begin{aligned}
\mu_{K(\varepsilon)}(x) & =\max \left\{\mu_{H(\varepsilon)}(x), \mu_{F(\varepsilon)}(x)\right\} \\
& =\max \left\{\mu_{F(\varepsilon)}(x), \mu_{F(\varepsilon)}(x)\right\} \\
& =\mu_{F(\varepsilon)}(x)
\end{aligned}
$$

and

$$
\begin{aligned}
\nu_{K(\varepsilon)}(x) & =\min \left\{\nu_{H(\varepsilon)}(x), \nu_{F(\varepsilon)}(x)\right\} \\
& =\min \left\{\nu_{F(\varepsilon)}(x), \nu_{F(\varepsilon)}(x)\right\} \\
& =\nu_{F(\varepsilon)}(x) .
\end{aligned}
$$

Hence $(\langle F, A\rangle \sqcap\langle G, B\rangle) \tilde{\cup}\langle F, A\rangle=\langle F, A\rangle$.

Theorem 3.12. Let $\langle F, A\rangle,\langle G, B\rangle,\langle H, C\rangle \in \mathcal{G} \mathcal{I} \mathcal{F} \mathcal{S}(U$, $E)$, then

$$
\begin{aligned}
& \langle F, A\rangle \tilde{\cup}(\langle G, B\rangle \sqcap\langle H, C\rangle) \\
& =(\langle F, A\rangle \tilde{\cup}\langle G, B\rangle) \sqcap(\langle F, A\rangle \tilde{\cup}\langle H, C\rangle) .
\end{aligned}
$$

Proof. Suppose that $\langle F, A\rangle \tilde{\cup}(\langle G, B\rangle \sqcap\langle H, C\rangle)=\langle K, A \cap$ $(B \cup C)\rangle$ and $(\langle F, A\rangle \tilde{\cup}\langle G, B\rangle) \sqcap(\langle F, A\rangle \tilde{\cup}\langle H, C\rangle)=$ $\langle L,(A \cap B) \cup(A \cap C)\rangle=\langle L, A \cap(B \cup C)\rangle$.

For all $\varepsilon \in A \cap(B \cup C)$ and $x \in U$, (a) if $\varepsilon \in A, \varepsilon \notin B, \varepsilon \in C$, then

$$
\begin{aligned}
\mu_{K(\varepsilon)}(x) & =\max \left\{\mu_{F(\varepsilon)}(x), \mu_{H(\varepsilon)}(x)\right\} \\
& =\mu_{L(\varepsilon)}(x)
\end{aligned}
$$

and

$$
\begin{aligned}
\nu_{K(\varepsilon)}(x) & =\min \left\{\nu_{F(\varepsilon)}(x), \nu_{H(\varepsilon)}(x)\right\} \\
& =\nu_{L(\varepsilon)}(x) .
\end{aligned}
$$

(b) if $\varepsilon \in A, \varepsilon \in B, \varepsilon \notin C$, then

$$
\begin{aligned}
\mu_{K(\varepsilon)}(x) & =\max \left\{\mu_{F(\varepsilon)}(x), \mu_{G(\varepsilon)}(x)\right\} \\
& =\mu_{L(\varepsilon)}(x)
\end{aligned}
$$

and

$$
\begin{aligned}
\nu_{K(\varepsilon)}(x) & =\min \left\{\nu_{F(\varepsilon)}(x), \nu_{G(\varepsilon)}(x)\right\} \\
& =\nu_{L(\varepsilon)}(x) .
\end{aligned}
$$

(c) if $\varepsilon \in A, \varepsilon \in B, \varepsilon \in C$, then

$$
\begin{aligned}
\mu_{K(\varepsilon)}(x)= & \max \left\{\mu_{F(\varepsilon)}(x), \min \left\{\mu_{G(\varepsilon)}(x), \mu_{H(\varepsilon)}(x)\right\}\right\} \\
= & \min \left\{\max \left\{\mu_{F(\varepsilon)}(x), \mu_{G(\varepsilon)}(x)\right\}\right. \\
& \left.\max \left\{\mu_{F(\varepsilon)}(x), \mu_{H(\varepsilon)}(x)\right\}\right\} \\
= & \mu_{L(\varepsilon)}(x)
\end{aligned}
$$

and

$$
\begin{aligned}
\nu_{K(\varepsilon)}(x)= & \min \left\{\nu_{F(\varepsilon)}(x), \max \left\{\nu_{G(\varepsilon)}(x), \nu_{H(\varepsilon)}(x)\right\}\right\} \\
= & \max \left\{\min \left\{\nu_{F(\varepsilon)}(x), \nu_{G(\varepsilon)}(x)\right\}\right. \\
& \left.\min \left\{\nu_{F(\varepsilon)}(x), \nu_{H(\varepsilon)}(x)\right\}\right\} \\
= & \nu_{L(\varepsilon)}(x)
\end{aligned}
$$


Hence $\langle F, A\rangle \tilde{\cup}(\langle G, B\rangle \sqcap\langle H, C\rangle)=(\langle F, A\rangle \tilde{\cup}\langle G, B\rangle) \sqcap$ $(\langle F, A\rangle \tilde{\cup}\langle H, C\rangle)$.

Theorem 3.13. (1) $(\mathcal{G I F \mathcal { S }}(U, E), \tilde{\cup}, \sqcap)$ is a distributive lattice.

(2) Let $\leq_{2}$ be the ordering relation in lattice $(\mathcal{G I F \mathcal { S }}(U, E), \tilde{\cup}, \sqcap)$ and $\langle F, A\rangle,\langle G, B\rangle \in \mathcal{G} \mathcal{I} \mathcal{F} \mathcal{S}(U, E)$. Then $\langle F, A\rangle \leq_{2}\langle G, B\rangle$ if and only if $B \subseteq A$, and $\mu_{F(\varepsilon)}(x) \leq \mu_{G(\varepsilon)}(x)$ and $\nu_{F(\varepsilon)}(x) \geq \nu_{G(\varepsilon)}(x)$ for all $\varepsilon \in B$ and $x \in U$.

Proof. (1) This can be deduced from Theorem 3.3, 3.11 and 3.12.

(2) Suppose that $\langle F, A\rangle \leq_{2}\langle G, B\rangle$. Then $\langle F, A\rangle \tilde{\cup}$ $\langle G, B\rangle=\langle G, B\rangle$. So, by definition, we have $A \cap$ $B=B$ and hence $B \subseteq A$. For all $\varepsilon \in B$ and $x \in U$, since $\max \left\{\mu_{F(\varepsilon)}(x), \mu_{G(\varepsilon)}(x)\right\}=\mu_{G(\varepsilon)}(x)$ and $\min \left\{\nu_{F(\varepsilon)}(x), \nu_{G(\varepsilon)}(x)\right\}=\nu_{G(\varepsilon)}(x)$, we have $\mu_{F(\varepsilon)}(x) \leq \mu_{G(\varepsilon)}(x)$ and $\nu_{F(\varepsilon)}(x) \geq \nu_{G(\varepsilon)}(x)$.

Conversely, suppose that $B \subseteq A$, and $\mu_{F(\varepsilon)}(x) \leq$ $\mu_{G(\varepsilon)}(x)$ and $\nu_{F(\varepsilon)}(x) \geq \nu_{G(\varepsilon)}(x)$ for all $\varepsilon \in B$ and $x \in U$. It is trivial to verify that $\langle F, A\rangle \tilde{\cup}\langle G, B\rangle=\langle G, B\rangle$ and hence $\langle F, A\rangle \leq_{2}\langle G, B\rangle$.

Corollary 3.14. $\mathcal{G} \mathcal{I} \mathcal{F} \mathcal{S}_{A}$ is sublattice of $(\mathcal{G I F S}(U, E)$, $\tilde{U}, \sqcap)$.

In what follows, we discuss the properties of restricted union and restricted intersection operations related to necessity and possibility operations.

Definition 3.15. [16] (1) The necessity operation on a generalized intuitionistic fuzzy soft set $\langle F, A\rangle$ is denoted by $\odot\langle F, A\rangle$ and is defined as $\odot\langle F, A\rangle=$ $\left\{\left\langle x, \mu_{\odot F(\varepsilon)}(x), \nu_{\odot F(\varepsilon)}(x)\right\rangle: x \in U, \varepsilon \in A\right\}$, where $\mu_{\odot F(\varepsilon)}(x)=\mu_{F(\varepsilon)}(x)$ and $\nu_{\odot F(\varepsilon)}(x)=1-\mu_{F(\varepsilon)}(x)$.

(2) The possibility operation on a generalized intuitionistic fuzzy soft set $\langle F, A\rangle$ is denoted by $\diamond\langle F, A\rangle$ and is defined as $\diamond\langle F, A\rangle=\left\{\left\langle x, \mu_{\diamond F(\varepsilon)}(x), \nu_{\diamond F(\varepsilon)}(x)\right\rangle\right.$ : $x \in U, \varepsilon \in A\}$, where $\mu_{\diamond F(\varepsilon)}(x)=1-\nu_{F(\varepsilon)}(x)$ and $\nu_{\diamond F(\varepsilon)}(x)=\nu_{F(\varepsilon)}(x)$.

Theorem 3.16. Let $\langle F, A\rangle$ and $\langle G, B\rangle$ be two generalized intuitionistic fuzzy soft sets over a universe $U$, then

(1) $\odot(\langle F, A\rangle \tilde{\cup}\langle G, B\rangle)=\odot\langle F, A\rangle \tilde{\cup} \odot\langle G, B\rangle$;

(2) $\odot(\langle F, A\rangle \tilde{\cap}\langle G, B\rangle)=\odot\langle F, A\rangle \tilde{\cap} \odot\langle G, B\rangle$.
Proof. (1) Suppose that $\langle F, A\rangle \tilde{\cup}\langle G, B\rangle=\langle H, A \cap B\rangle$ and $\odot(\langle F, A\rangle \tilde{\cup}\langle G, B\rangle)=\odot\langle H, A \cap B\rangle$. For all $\varepsilon \in A \cap B$ and $x \in U$, we have

$$
\begin{aligned}
& \mu_{\odot H(\varepsilon)}(x)=\max \left\{\mu_{F(\varepsilon)}(x), \mu_{G(\varepsilon)}(x)\right\}, \\
& \nu_{\odot H(\varepsilon)}(x)=1-\max \left\{\mu_{F(\varepsilon)}(x), \mu_{G(\varepsilon)}(x)\right\} .
\end{aligned}
$$

Assume that $\odot\langle F, A\rangle=\left\{\left\langle x, \mu_{F(\varepsilon)}(x), 1-\mu_{F(\varepsilon)}(x)\right\rangle\right.$ : $x \in U, \varepsilon \in A\}$ and $\odot\langle G, B\rangle=\left\{\left\langle x, \mu_{G(\varepsilon)}(x), 1-\right.\right.$ $\left.\left.\mu_{G(\varepsilon)}(x)\right\rangle: x \in U, \varepsilon \in B\right\}$. Suppose that $\odot\langle F, A\rangle \tilde{\cup} \odot$ $\langle G, B\rangle=\langle O, A \cap B\rangle$. For all $\varepsilon \in A \cap B$ and $x \in U$,

$$
\mu_{O(\varepsilon)}(x)=\max \left\{\mu_{F(\varepsilon)}(x), \mu_{G(\varepsilon)}(x)\right\}
$$

and

$$
\begin{aligned}
\nu_{O(\varepsilon)}(x) & =\min \left\{1-\mu_{F(\varepsilon)}(x), 1-\mu_{G(\varepsilon)}(x)\right\} \\
& =1-\max \left\{\mu_{F(\varepsilon)}(x), \mu_{G(\varepsilon)}(x)\right\} .
\end{aligned}
$$

Hence $\odot(\langle F, A\rangle \tilde{\cup}\langle G, B\rangle)=\odot\langle F, A\rangle \tilde{\cup} \odot\langle G, B\rangle$.

(2) is proved analogously.

Theorem 3.17. Let $\langle F, A\rangle$ and $\langle G, B\rangle$ be two generalized intuitionistic fuzzy soft sets over a universe $U$, then

(1) $\diamond(\langle F, A\rangle \tilde{\cup}\langle G, B\rangle)=\diamond\langle F, A\rangle \tilde{\cup} \diamond\langle G, B\rangle$;

(2) $\diamond(\langle F, A\rangle \tilde{\cap}\langle G, B\rangle)=\diamond\langle F, A\rangle \tilde{\cap} \diamond\langle G, B\rangle$.

Proof. (1) Suppose that $\langle F, A\rangle \tilde{\cup}\langle G, B\rangle=\langle H, A \cap B\rangle$ and $\diamond(\langle F, A\rangle \tilde{\cup}\langle G, B\rangle)=\diamond\langle H, A \cap B\rangle$. For all $\varepsilon \in A \cap B$ and $x \in U$, we have

$$
\begin{aligned}
& \mu_{\diamond H(\varepsilon)}(x)=1-\min \left\{\nu_{F(\varepsilon)}(x), \nu_{G(\varepsilon)}(x)\right\}, \\
& \nu_{\diamond H(\varepsilon)}(x)=\min \left\{\nu_{F(\varepsilon)}(x), \nu_{G(\varepsilon)}(x)\right\} .
\end{aligned}
$$

Assume that $\diamond\langle F, A\rangle=\left\{\left\langle x, 1-\nu_{F(\varepsilon)}(x), \nu_{F(\varepsilon)}(x)\right\rangle: x \in\right.$ $U, \varepsilon \in A\}$ and $\diamond\langle G, B\rangle=\left\{\left\langle x, 1-\nu_{G(\varepsilon)}(x), \nu_{G(\varepsilon)}(x)\right\rangle\right.$ : $x \in U, \varepsilon \in B\}$. Suppose that $\diamond\langle F, A\rangle \tilde{\cup} \diamond\langle G, B\rangle=$ $\langle O, A \cap B\rangle$ For all $\varepsilon \in C$ and $x \in U$,

$$
\begin{aligned}
\mu_{O(\varepsilon)}(x) & =\max \left\{1-\nu_{F(\varepsilon)}(x), 1-\nu_{G(\varepsilon)}(x)\right\} \\
& =1-\min \left\{\nu_{F(\varepsilon)}(x), \nu_{G(\varepsilon)}(x)\right\}
\end{aligned}
$$

and

$$
\nu_{O(\varepsilon)}(x)=\min \left\{\nu_{F(\varepsilon)}(x), \nu_{G(\varepsilon)}(x)\right\} .
$$

Hence $\diamond(\langle F, A\rangle \tilde{\cup}\langle G, B\rangle)=\diamond\langle F, A\rangle \tilde{\cup} \diamond\langle G, B\rangle$.

(2) is proved analogously. 


\section{Conclusions}

Generalized intuitionistic fuzzy soft set theory has been regarded as an effective mathematical tool to deal with uncertainties. In this paper, we define the restricted union and restricted intersection operations on the generalized intuitionistic fuzzy soft sets. The basic properties of the operations and their interconnections between each other are presented and discussed. We deal with the algebraic structure of generalized intuitionistic fuzzy soft sets. The lattice structures of generalized intuitionistic fuzzy soft sets are constructed.

\section{References}

[1] D. Molodtsov, "Soft set theory - first results," Computers and Mathematics with Applications, vol. 37, no. 4-5, pp. 19-31, 1999.

[2] M.I. Ali, F. Feng, X. Liu, W.K. Min and M. Shabir, "On some new operations in soft set theory," Computers and Mathematics with Applications, vol. 57, no. 9, pp. 1547-1553, 2009.

[3] Z. Kong, L. Gao and L. Wang, "Comment on "A fuzzy soft set theoretic approach to decision making problems," Journal of Computational and Applied Mathematics, vol. 223, no. 2, pp. 540-542, 2009.

[4] P.K. Maji, R. Biswas and A.R. Roy, "Fuzzy soft sets," Journal of Fuzzy Mathematics, vol. 9, no. 3, pp. 589602, 2001.

[5] P.K. Maji, R. Biswas and A.R. Roy, "Intuitionistic fuzzy soft sets," Journal of Fuzzy Mathematics, vol. 9, no. 3, pp. 677-692, 2001.

[6] P.K. Maji, R. Biswas and A.R. Roy, "Soft set theory," Computers and Mathematics with Applications, vol. 45, no. 4-5, pp. 555-562, 2003.

[7] P.K. Maji, A.R. Roy and R. Biswas, "On intuitionistic fuzzy soft sets," Journal of Fuzzy Mathematics, vol. 12, no. 3, pp. 669-683, 2004.

[8] P.K. Maji, More on intuitionistic fuzzy soft sets. In: H. Sakai, M.K. Chakraborty, A.E. Hassanien, D. Slezak and W. Zhu, Editors, Proceedings of the 12th International Conference on Rough Sets, Fuzzy Sets, Data
Mining and Granular Computing RSFDGrC 2009, Lecture Notes in Computer Science, vol. 5908, pp. 231-240, 2009.

[9] P. Majumdar and S.K. Samanta, "Generalised fuzzy soft sets," Computers and Mathematics with Applications, vol. 59, no. 4, pp. 1425-1432, 2010.

[10] J.H. Park, Y.C. Kwun and M.J. Son, "A generalized intuitionistic fuzzy soft set theoretic approach to decision making problems," International Journal of Fuzzy Logic and Intelligent Systems, vol. 11, no. 2, pp. 71-76, 2011.

[11] A.R. Roy and P.K. Maji, "A fuzzy soft set theoretic approach to decision making problems," Journal of Computational and Applied Mathematics, vol. 203, no. 2, pp. 412-418, 2007.

[12] Y. Zou and Z. Xiao, "Data analysis approaches of soft sets under incomplete information," KnowledgeBased Systems, vol. 21, no. 8, pp. 941-945, 2008.

[13] W. Xu, J. Ma, S. Wang and G. Hao, "Vague soft sets and their properties," Computers and Mathematics with Applications, vol. 59, no. 2, pp. 787-794, 2010.

[14] J.H. Park, Y.C. Kwun and J.S. Hwang, "Generalized intuitionistic fuzzy soft sets," Journal of Korean Institute of Intelligent Systems, vol. 21, no. 3, pp. 389-394, 2011.

[15] T.K. Mondal and S.K. Samanta, "Generalized intuitionistic fuzzy sets," Journal of Fuzzy Mathematics, vol. 10, pp. 839-861, 2002.

[16] J.H. Park, "On operations on generalized intuitionistic fuzzy soft sets," International Journal of Fuzzy Logic and Intelligent Systems, vol. 11, no. 3, pp. 184189, 2011.

[17] J.H. Park, K.M. Lim and B.Y. Lee, "Correlation coefficient between generalized Atanassov's intuitionistic fuzzy sets," International Journal of Uncertainty, Fuzziness \& Knowledge-Based Systems, vol. 17, no. 3, pp. 449-461, 2009. 
Journal of Korean Institute of Intelligent Systems, Vol. 24, No. 2, April 2014

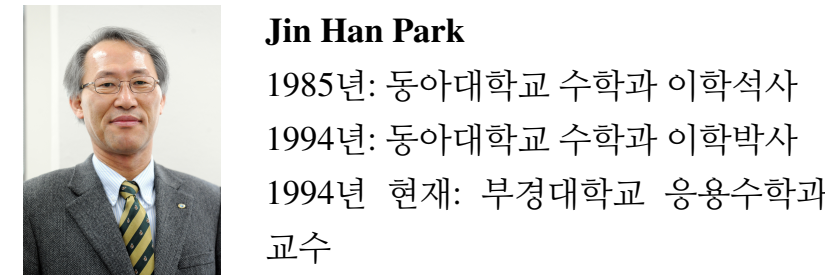

2003년 현재: 한국지능시스템학회 이사

2011년: 한국지능시스템학회 학술상

관심분야 : Fuzzy topology, Decision making

Phone : +82-10-2625-6326

E-mail : jihpark@pknu.ac.kr 